\title{
Elaboration de composite cimentaire à base de diss « Ampelodesma Mauritanica ॥
}

\author{
MERZOUD Mouloud* et HABITA Mohamed Fouzi \\ Laboratoire de Génie Civil, Université Badji Mokhtar, B.P I2 Annaba, Algérie
}

*Correspondance,courriel : merzoud mouloud@yahoo.fr

\section{Résumé}

Le Diss (Ampelodesmos mauritanicus, famille des Poacées) est une grande graminée répandue dans l'Afrique-du-Nord méditerranéenne et les régions sèches de la Grèce à l'Espagne. En France, on la trouve dans les départements des Alpes-Maritimes, du Var, de la Corse-du-Sud et de l'Hérault. Cette plante était utilisée auparavant dans la réalisation des habitations anciennes de ces régions en raison de ses qualités mécaniques et hydriques.

L'utilisation d'une telle plante fibreuse dans une pâte cimentaire offre des résistances aux tractions très intéressantes, qui font de ce matériau léger un excellent remplissage pour les structures soumises aux efforts sismiques.

L'objectif de nos travaux était l'élaboration des composites à base de diss dans des matrices cimentaires.

Le matériau végétal de base, très fibreux, présente une absorption de l'ordre de $90 \%$. L'utilisation des fibres de diss naturel broyé comme agrégat dans des matrices cimentaires présente un retard de prise assez considérable et des résistances très faibles, alors que les fibres présentent des résistances à la tension considérable. Pour améliorer la contribution des fibres dans les composites à base cimentaire, nous avons donc procédé au traitement par ébullition des fibres de diss pour extraire les substances causes de la mauvaise liaison entre les fibres et la pâte de ciment. Nous avons constaté que le traitement à l'eau bovillie a été très efficace, et a bien amélioré les propriétés mécaniques du composite.

Mots-clés : Diss, Ampelodesma mauritanica, fibres lignocellulosiques, déchets de bois, composites. 


\section{Abstract \\ Developpement of cementious composite based on a Diss (Ampelodesmos mauritanicus)}

The Diss (Ampelodesmos mauritanicus, family of Poaceae) is a very luxuriant plant growing in wild state around the Mediterranean North Africa and dry areas of Greece and Spain. It grows in France, mainly, in the departments of the Alpes-Maritimes, the Var, the South of Corsica and Herault. In the past, it was used as building material because of its mechanical and hydrous qualities.

The use of such a fibrous plant in a cementitious matrix leads to lightweight materials with very attractive tensile behavior that can be used as advantageous filling materials for structures subjected to seismic effects.

This paper is focused on the optimisation of this kind of material on the basis of mechanical properties.

The basic vegetable material, very fibrous, presents indeed an absorption of about $90 \%$ that would be corrected. Moreover, we noted a considerable retardation of setting and very low resistances during the composite tests with natural crushed diss, despite the fact that the fibres have considerable tensile strength. To improve the fibres contribution in cementitious composites, we have carried out a treatment by boiling the fibres of diss to extract the substances responsible for the bad connection between fibres and the cement paste. The results obtained are encouraging and enable to foresee a later development of this material.

\section{Keywords : Diss, Ampelodesma mauritanica, lignocellulosic fibres, chips of wood, composites}

\section{Introduction}

Les matériaux végétaux représentent une source de produits renouvelables utilisés ces dernières décennies dans la construction. Le matériau diss étant fibreux, et résistant à la traction, offre au composite une ductilité comparable à celle d'un béton à fibres classiques, alors que la masse volumique est beaucoup plus réduite.

Le manque de bibliographie sur les fibres de diss, confirme sa première utilisation dans la fabrication de composites à base cimentaire. Néanmoins, Savastano Jr H et al [l] ont utilisé des résidus de sisal, de bananier et d'eucalyptus comme renfort dans des composites cimentaires. Les composites ainsi obtenus présentent des performances mécaniques acceptables. Les travaux de Kriker $A$ et al [2], basés sur l'utilisation de quatre types de fibres de palmier dattier dans une matrice cimentaire, ont montré que 
l'augmentation des longueurs et des pourcentages des fibres améliorent la résistance à la flexion et la dureté du composite, mais diminue les résistances à la compression. Ledhem A et al [3] ont montré lors de leurs travaux sur composites cimentaires à base de copeaux de bois que le traitement thermique du bois pouvait augmenter les résistances mécaniques, la conductivité thermique, ainsi les variations dimensionnelles extrêmes des composites. Aamar Daya E.H [4] a utilisé des poussières issues du teillage des fibres de lin comme agrégat dans un composite à matrice cimentaire. Ses travaux ont montré que le traitement à l'eau bouillie des poussières du lin améliore considérablement les résistances mécaniques des composites.

Toutefois la présence d'un végétal au sein d'une matrice cimentaire peut faire appréhender une sensibilité accrue à l'eau : outre des désordres mécaniques, des baisses de performances mécaniques et surtout thermiques sont également à craindre (Piementa et al [5]). Le milieu fortement alcalin développé par l'hydratation du ciment provoque en effet des réactions d'hydrolyse et solubilise certains composés comme les sucres, les hémicelluloses et les pectines (Simatupang [6]). Garci Juenger M.C et al [7] et Bilba K et al [8] ont étudié l'influence des sucres sur la prise des composites cimentaires et ont montré que le sucre retarde l'hydratation du ciment.

Des fibres de noix de coco ont fait également l'objet d'une étude menée par Asasutjarit C et al [9] pour l'élaboration d'un composite cimentaire à usage de panneaux isolants résistants. Ils ont étudié principalement les paramètres relatifs à la dimension optimale des fibres, leur prétraitement et les pourcentages des éléments constituants le composite. Ils ont trouvé que les dimensions optimales des fibres varient entre 1 à $6 \mathrm{~cm}$, que le traitement à l'eau bouillie et le lavage des fibres améliore les caractéristiques mécaniques, et que la formulation optimale en poids de (ciment : fibre : eau) est $2: 1$ :2. La durabilité des bétons légers à base de résidus de bois ont fait été étudiés par Coatanlem P [10], ils ont trouvés que les résidus saturés dans une dolution de silicates de sodium améliore les caractéristiques mécaniques, à cause de la bonne adhésion des résidus de bois et la pâte de ciment.

Les composites à base de fibres naturelles ont vu un développement important pour leurs caractéristiques mécaniques intéressantes, leur pouvoir isolant, et surtout pour le prix de revient intéressant. Des études systématiques sont à prévoir pour chaque type de matériau et son utilisation dans le domaine du bâtiment [11].

L'étude de la littérature concernant les fibres de Diss semble prouver un manque de valorisation technologique, notamment dans le domaine des composites cimentaires. 0 r, cette espèce végétale existe à l'état sauvage en quantité importante sur le pourtour méditerranéen et sa nature fibreuse est susceptible d'offrir aux matériaux cimentaires les qualités apportées par les fibres classiques. 
L'effet de la présence de substances hydrosolubles dans la partie lignocellulosique qui affecte la réaction d'hydratation du ciment, qui provoque un retard de la prise et du durcissement du composite a été réduit par l'ébullition des fibres de diss.

\section{Matériaux et méthodes expérimentales:}

\subsection{Matériaux :}

Le matériau diss utilisé comme granulat dans nos composites, a été broyé avec un broyeur à couteaux à découpe parallèle de type Retsch, avec une maille de $10 \mathrm{~mm}$. Après le broyage, nous avons considéré les fibres comme suit :

- fibres de diss naturel, séchées à l'étuve

- fibres traitées thermiquement à l'eau bouillante, puis séchées à l'étuve Lors du traitement à l'eau bouillie, nous avons conservé le résidu d'eau d'ébullition pour étudier d'éventuels effets sur la prise du ciment.

Le ciment utilisé est de type CPA CEMI 52.5 (NF P 15-301.

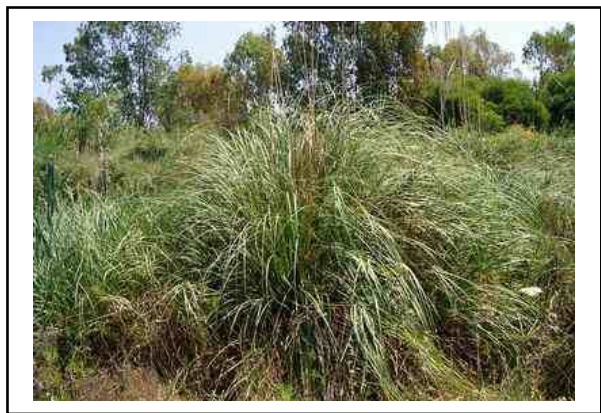

Figure 1 : Plante de diss

(ampelodesma mauritanica)

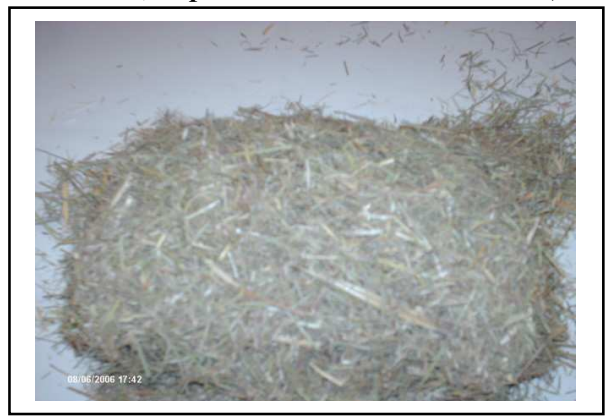

Figure 3 : Fibres de diss broyées

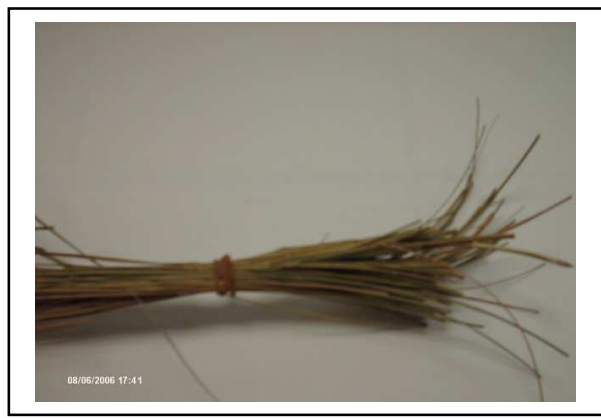

Figure 2 : Tiges de diss

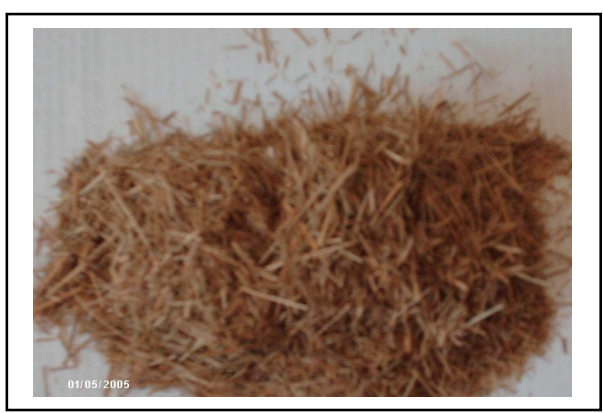

Figure 4 : Fibres de Diss broyées et bouillies 


\subsection{Méthodes expérimentales:}

La morphologie des différentes fibres a été étudiée par microscopie électronique à balayage (MEB).

Les images ont été réalisées à l'aide d'un :

- vidéo microscope (Controlabß) VH-Z25 muni d'un zoom 25x à 175x,

- un éclairage annulaire à source de lumière froide, non diffus, semi-rasant, positionné sur la base du vidéo microscope, permettant la mise en évidence du relief des échantillons,

- un écran haute résolution 507X688 pixels,

- un PC,

- un système de vision VIDEOMET (Controlabß) permettant la numérisation et la visualisation images.

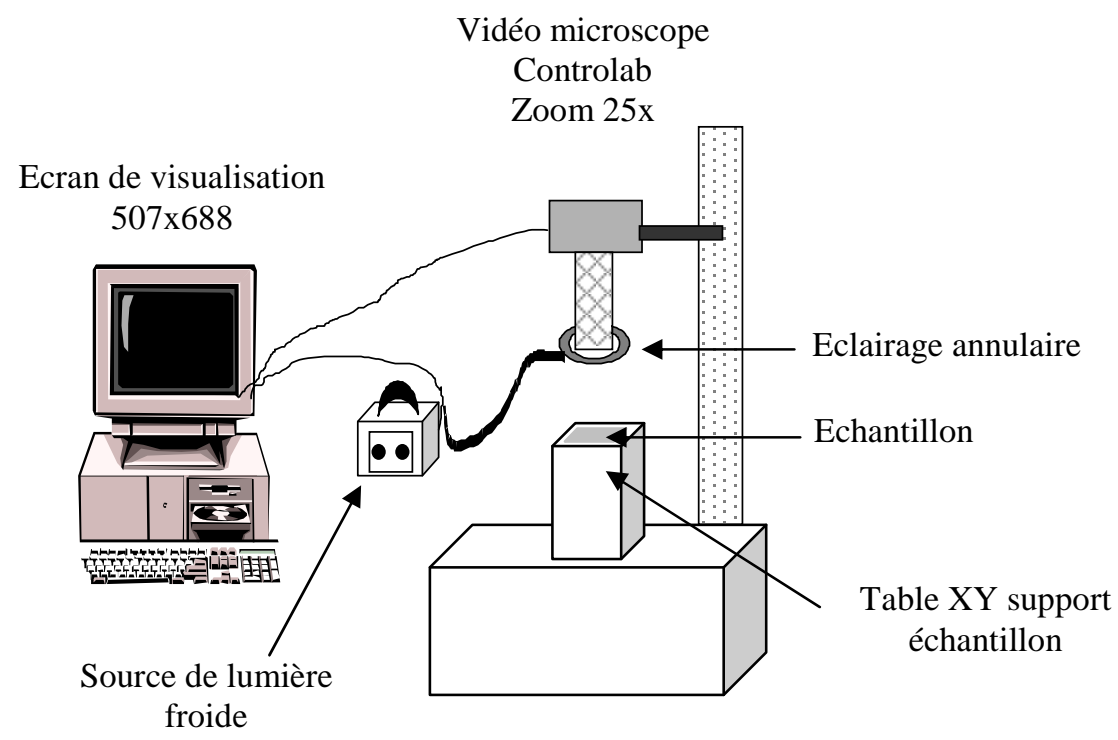

Figure. 5 : Dispositif d'analyse d'images

Les éprouvettes confectionnées avec différentes fibres, traitées ou non, sont conservées pendant 28 jours dans une chambre humide (R.H $=95 \%, t=20^{\circ} \mathrm{C}$, puis placées à l'étuve à $50^{\circ} \mathrm{C}$ jusqu'à masse constante.

Les essais d'absorption ont été déterminés en immergeant une quantité de diss préalablement séchée et pesée $M(0)$. Les fibres sont enveloppées d'une toile fine pour éviter toute perte des fibres. Des pesées sont effectuées à des temps d'immersion 
définis $M(t)$, après essorage de l'échantillon pour enlever l'eau adsorbée . L'essai est suivi jusqu'à stabilisation de la masse absorbée. Le taux d'absorption en fonction de la racine du temps est déterminé par la relation :

$$
w(\%)=[M(t)-M(0)]^{*} 100 / M(0)
$$

Les résistances mécaniques en compression et en traction par flexion ont été déterminées, suivant la norme EN 196-1, sur des éprouvettes prismatiques $4 \times 4 \times 16$ $\mathrm{cm}$. Les essais de traction par flexion sont réalisés à l'aide d'un banc d'essai de flexion trois points, équipé d'un système d'acquisition. Les essais de compression sont réalisés sur la moitié des éprouvettes testées en flexion, à l'aide la machine type Perrier 68.7.

Les modules d'élasticité dynamiques ont été déterminés par auscultation sonique, type E0641 Ultrasonic Tester. Le principe est basé sur la détermination de la vitesse de propagation des ondes ultrasonores dans le matériau (célerité). Le module d'élasticité dynamique est donné par la relation : $\mathbf{E d}=\rho_{\mathrm{app}} \mathrm{C}_{\mathrm{L}}{ }^{2}$

Avec $\mathrm{Ed}=$ Module d'élasticité dynamique $(\mathrm{MPa}), \rho_{\text {app }}$ Masse volumique apparente de l'éprouvette $\left(\mathrm{kg} / \mathrm{m}^{3}\right), C_{L}=$ célérité de l'onde $(\mathrm{m} / \mathrm{s})$

\subsection{Formulations:}

Le Tableau I résume les pourcentages d'absorption des fibres végétales.

Tableau 1 : Pourcentage d'absorption des fibres de diss traitées et naurelles.

\begin{tabular}{|l|l|l|}
\hline Fibres végétales & \% d'absorption en eau & $\begin{array}{l}\text { Masse volumique } \\
\text { [grammes/litres] }\end{array}$ \\
\hline Non traitées et séchées & 92.38 & 50.3 \\
\hline Traitées à l'eau bouillie & 90.00 & 37.5 \\
\hline
\end{tabular}

Dans les formulations adoptées, nous avons fixé le rapport diss/ciment égal à 4.0 (en volume), en faisant varier le rapport Eau/Ciment en fonction du pourcentage d'absorption en eau des fibres végétales traitées ou non.

Lors de la confection des éprouvettes de diss non bouilli, nous avons constaté pour les composites un très grand retard de prise probablement dô à la présence d'essences extraites lors de l'ébullition des fibres naturelles. Dans cette partie du travail nous avons donc seulement utilisé du diss bouilli. Différents rapports eau/ciment soit $0.55,0.6,0.7$, 0.8 et 0.9 ont été considérés. La formulation à été optimisée sur la base de performances 
mécaniques. L'évolution des résistances mécaniques avec le rapport $\mathrm{E} / \mathrm{C}$ est donnée dans la Figure 6.

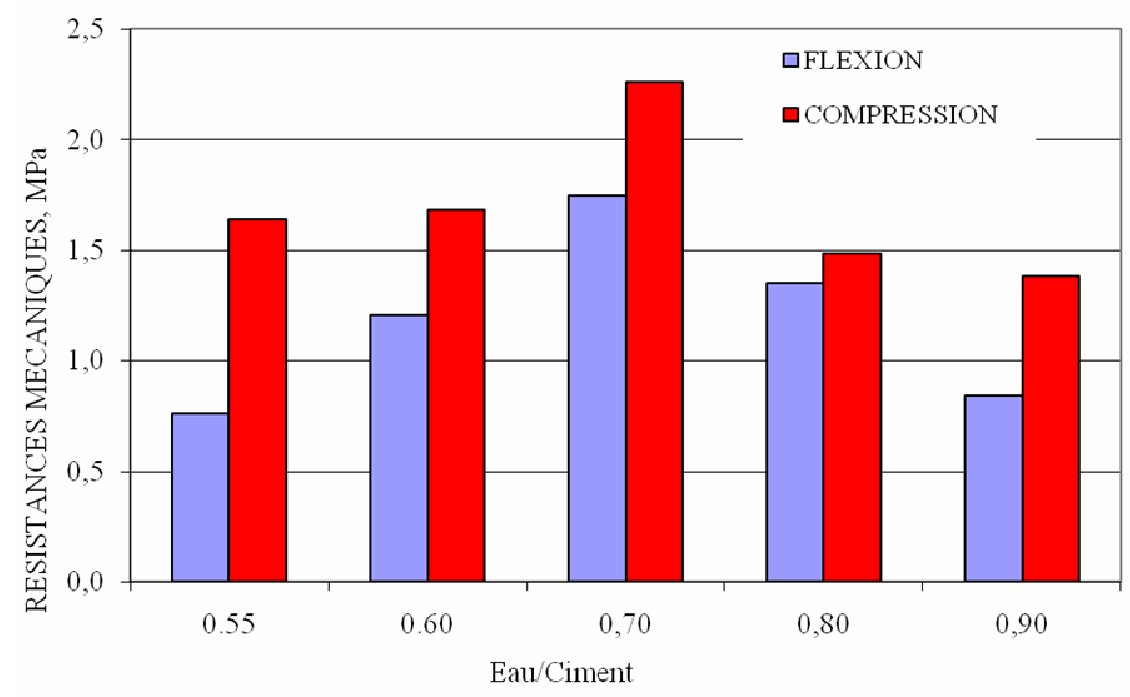

Figure $\mathbf{6}$ : Résistances à la traction et à la compression en fonction du rapport Eau/Ciment pour différentes formulations

Pour les formulations correspondant à $\mathrm{E} / \mathrm{C}=0.8$ et 0.9 , nous avons constaté lors du passage des éprouvettes à la table à choc une perte considérable de laitance, ce qui conduit à une erreur sur le rapport fibres/ciment.

Pour les rapports $\mathrm{E} / \mathrm{C}$ en dessous de $\mathbf{0 . 8}$, les fissures de traction par flexion au milieu de l'éprouvette n'ont pu avoir lieu qu'après le troisième chargement, et la rupture finale n'a pu avoir lieu qu'après le cinquième chargement.

Pour la suite de nos essais expérimentaux, nous avons préféré adopter pour le besoin de nos objectifs la formulation $4: 1: 0.7$, qui donne des résistances intéressantes, et des comportements plus ductiles que pour les autres formulations.

\section{3- Résultats et discussion}

\subsection{Modules d'élasticité dynamique}

Les valeurs des modules d'élasticité dynamique sont représentées par la Figure 7 en fonction des masses volumiques et pour les différentes formulations. 
Pour des masses volumiques du même ordre de grandeur, les valeurs des modules d'élasticité dynamiques sont très faibles pour les éprouvettes de diss non bouilli, à cause de l'absence d'adhérence des fibres de diss à la pâte de ciment en raison de la mauvaise hydratation du ciment. Elles sont par contre importantes pour les composites de diss traitées à l'eau bouillie.

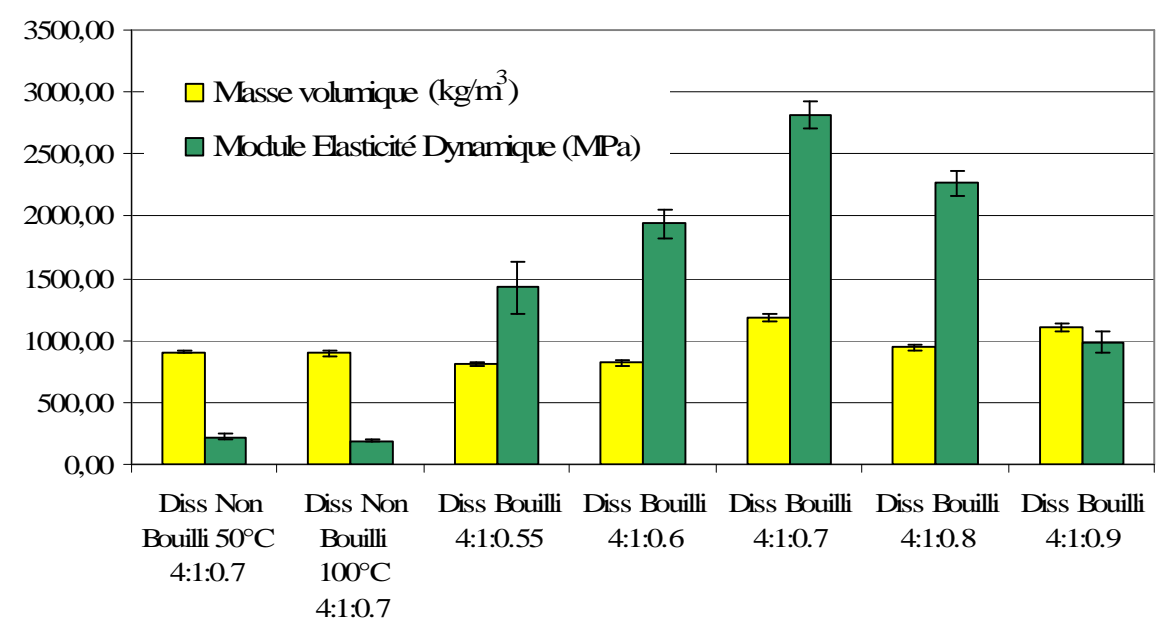

Figure 7 : Modules d'Elasticité dynamique et des masses volumiques pour différentes formulations

\section{2. Résistances mécaniques}

Les résistances à la traction par flexion et de compression sont représentées sur la Figure $\boldsymbol{8}$ en fonction des masses volumiques et pour différentes formulations.

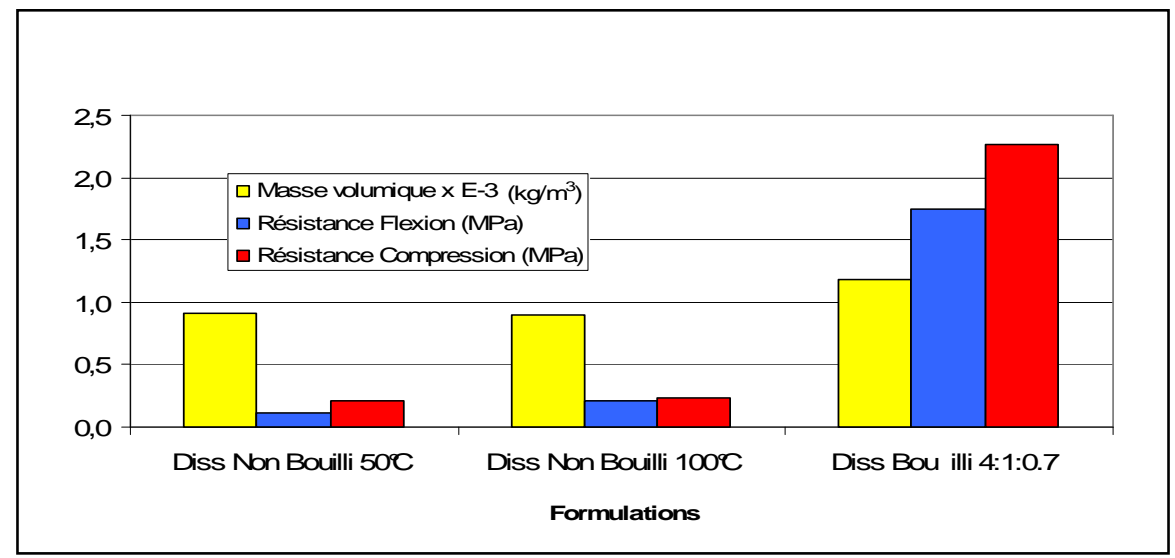

Figure 8: Résistances mécaniques et des masses volumiques pour différentes formulations

Mouloud MERZOUD et Mohamed Fouzi HABITA. 


\subsubsection{Cas de fibres végétales non traitées :}

Les résultats des résistances mécaniques des composites à base de diss non bouilli séché à l'étuve à 50 ou $100^{\circ} \mathrm{C}$ restent très faibles, malgré la présence d'épines qui réellement permettent une bonne adhésion des fibres à la pâte de ciment (voir Figure 9). Ce phénomène est certainement lié aux échanges qui peuvent se produire à l'interface matrice-granulat et aux réactions d'hydrolyse et de solubilisation de certains composés comme les sucres, les hémicelluloses et les pectines provoquées par le milieu fortement alcalin développé par l'hydratation du ciment. La présence de ces fractions solubles a été également confirmée par les essais d'adsorption en phase vapeur du diss. Au cours de ces derniers une moisissure des fibres de diss non bouilli a été observée au bout de onze jours alors qu'aucun développement n'a été constaté dans les fibres de diss bouilli pendant toute la période de l'essai (voir Figure 19. On peut donc penser que ce sont surtout ces fractions hydrosolubles qui entraînent la diminution de la cohésion des matériaux. C'est surtout l'existence de substances inhibitrices dans le composite qui diminuent la cohésion des matériaux pendant toute la période de la cure. L'image au vidéo microscope de la Figure 11 montre bien le manque de liaison lié au défaut d'hydratation.

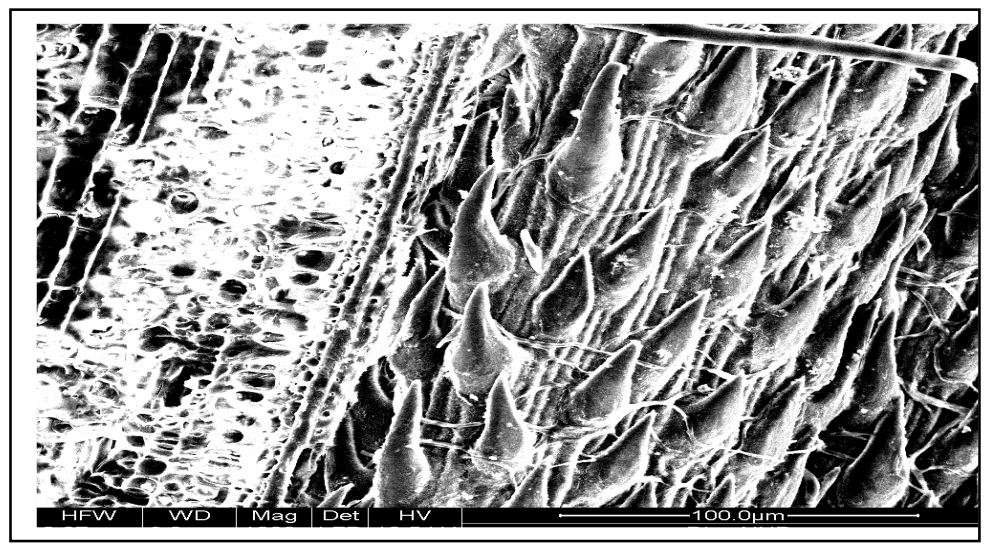

Figure 9 : Images au Microscope Electronique a Balayage d'une fibre de diss non bovilli, agrandissement $x 1000$ 


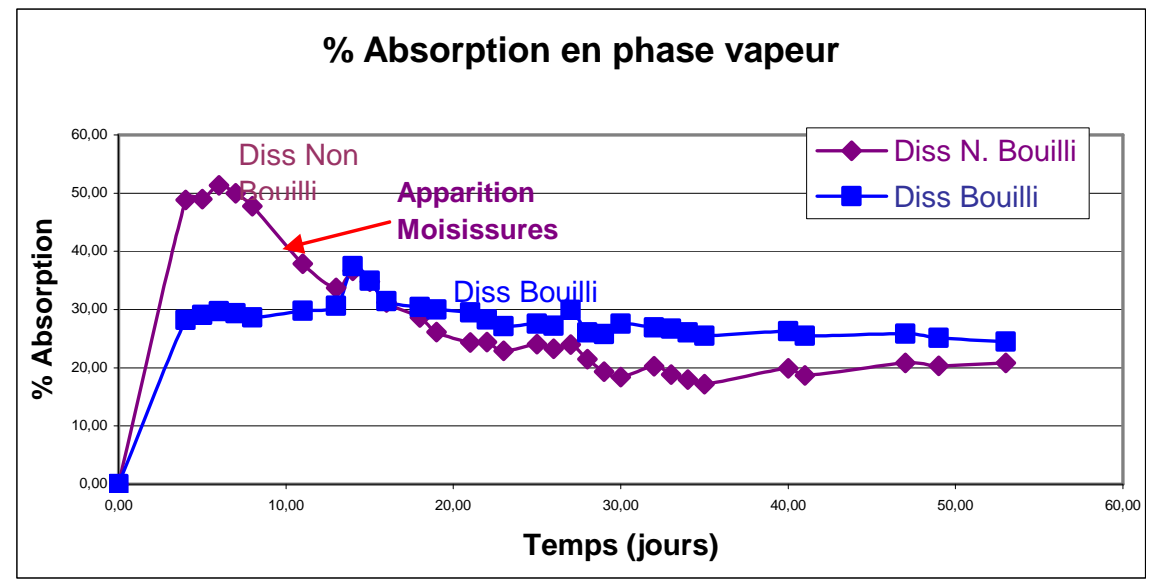

Figure 10 : Pourcentage d'adsorption en phase vapeur du diss bovilli et non bovilli

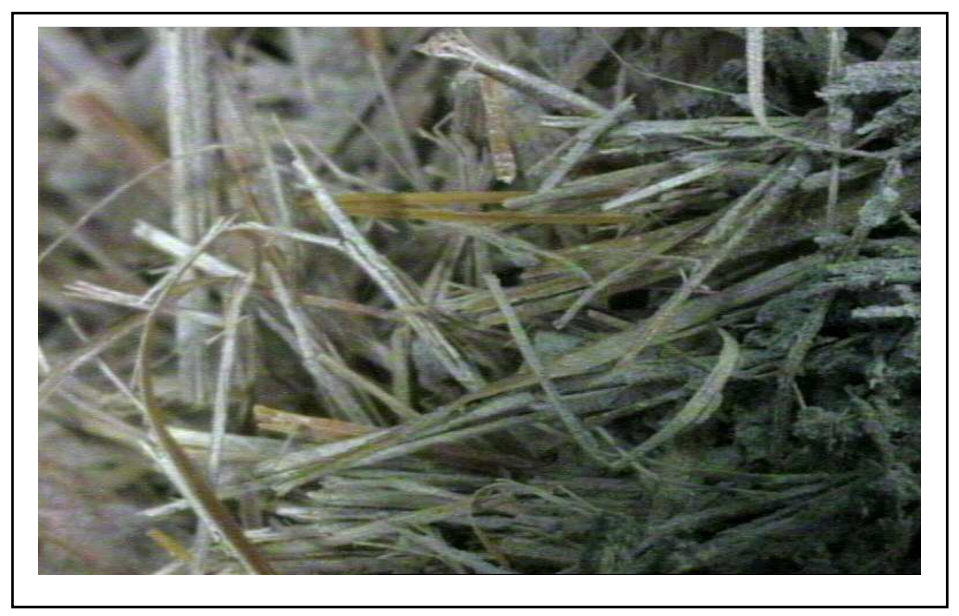

Figure 11 : Image au niveau de la section cisaillée du composite à base de diss non bovilli, agrandissement $\times 25$

\subsubsection{Influence du traitement hydrothermique :}

- Traitement par ébullition :

Le traitement par ébullition du diss permet la réduction des matières solubles responsables des retards de prise et d'incompatibilité avec la pâte de ciment. Ceci a pu être vérifié par les essais de prise sur la pâte de ciment en utilisant d'une part l'eau du réseau, et d'autre part l'eau d'extraction par ébullition. On a pu constater un retard de début prise considérable. Les résultats sont résumés sur le Tableau 2 
Tableau 2 : Essais de prise avec différentes eaux de gâchage

\begin{tabular}{|l|l|l|}
\hline Echantillon & Début de prise & Fin de prise \\
\hline Ciment+ Eau du réseau & $4 \mathrm{H} 30$ & $6 \mathrm{HOO}$ \\
\hline $\begin{array}{l}\text { Ciment+ Eau d'extraction de l'ébullition } \\
\text { du diss }\end{array}$ & $8 \mathrm{HOO}$ & $16 \mathrm{HOO}$ \\
\hline
\end{tabular}

Les fortes résistances ne sont pas dues essentiellement à la structure épineuse des fibres de diss qui permettent aux fibres de bien adhérer à la pâte de ciment, comme on le voit sur l'image prise au MEB de la Figure 12, mais surtout à la modification de la structure chimique des fractions végétales, notamment par la dégradation des hémicelluloses, en procédant au traitement thermique.

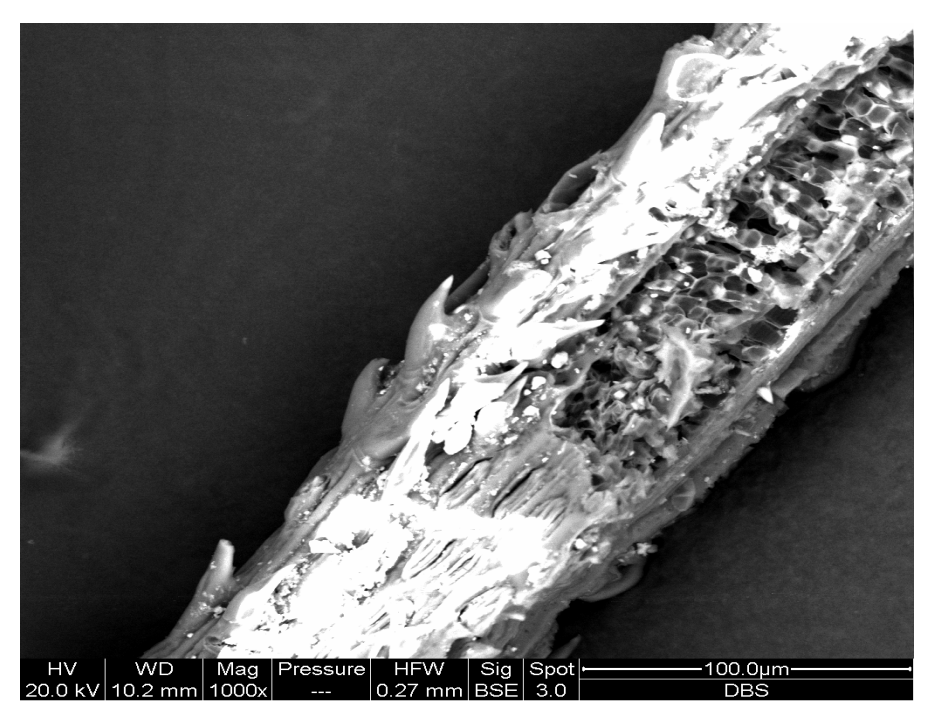

Figure 12 : Images au MEB des fibres de diss bouilli, agrandissements x 1000.

La résistance des composites cimentaires fibres est aussi liée au fait que les fibres se placent horizontalement, ce qui leur permet de bien adhérer à la pâte de ciment, et de jouer leur rôle en tant que renforcement. Ceci a été bien vérifié sur toutes les éprouvettes de composites à base de diss bouilli, où les ruptures ne sont pas fragiles, et on voit distinctement les zones comprimées et tendues, comme le montre la Figure $\mathbf{1 3}$. Cette augmentation de résistance est due au fait que les fibres de diss examinées au vidéo microscope (voir Figure 14 et 15 ) au niveau de la fissure par flexion de la partie tendue sont bien enrobées en paquets par la pâte de ciment, ce qui leur permet de bien résister aux efforts de traction qui leur sont appliquées. 


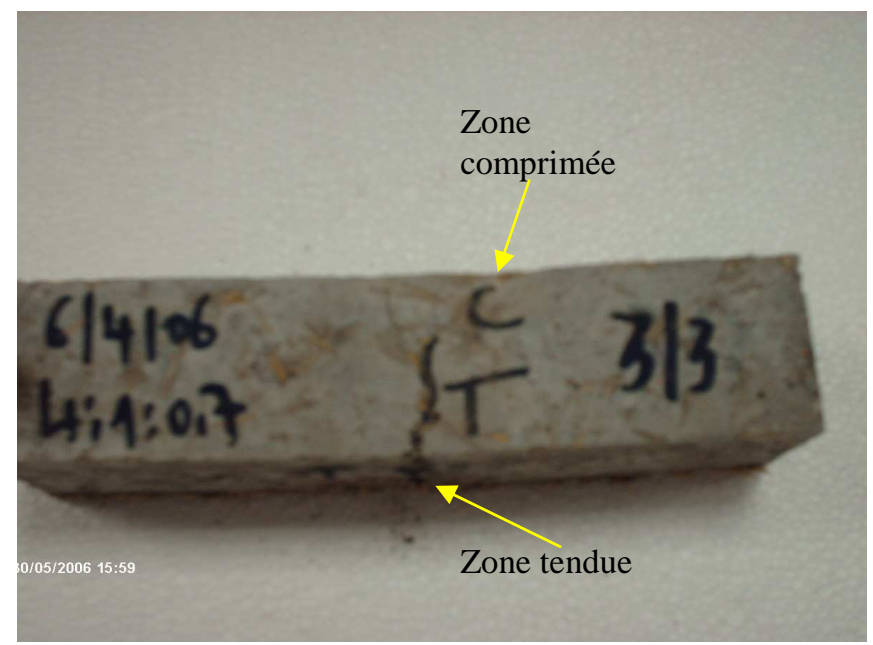

Figure 13 : Eprouvette à base de diss bouilli, formulation $4: 1: 0.7$, après essai de traction par flexion

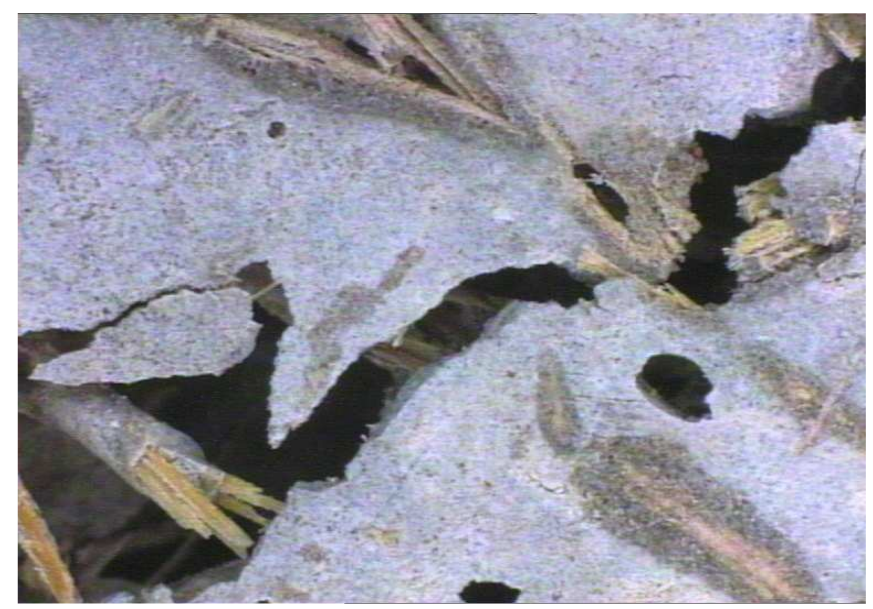

Figure 14 : Photo prise au vidéo microscope au niveau de la fissure du composite $\grave{a}$ base de fibres bovillies, agrandissement $\times 50$ 


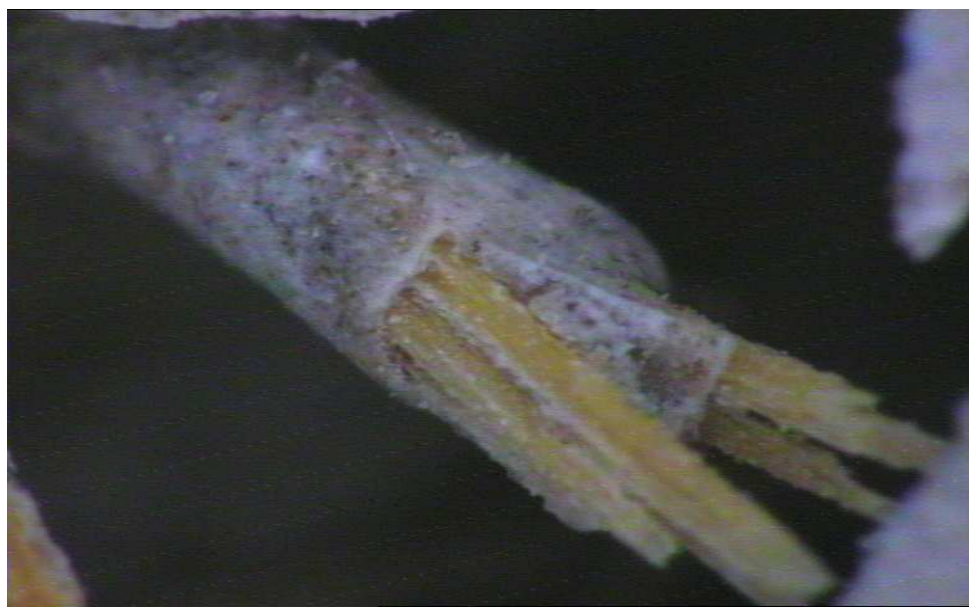

Figure 15 : Image au vidéo microscope des fibres à la partie tendue de l'éprouvette, du composite à base de diss bouilli, agrandissement $x$ I75

\subsection{Ruptures des éprouvettes en flexion et compression:}

Les éprouvettes de diss non bouilli n'ont manifesté aucune résistance, voir photo de la Figure 16, malgré la structure épineuse des fibres. Ceci est dô à la présence substances hydrosolubles dans la partie lignocellulosique qui affectent la réaction d'hydratation du ciment, ce qui provoque un retard de la prise et du durcissement du composite.

Lors des essais de flexion, nous avons constaté que les éprouvettes de diss bouilli n'ont manifesté aucune fissure à la première charge de rupture, et il aura fallu plusieurs cycles de chargement de moindres intensités pour aboutir à la rupture totale de l'éprouvette en flexion, et de même pour les essais de compression.

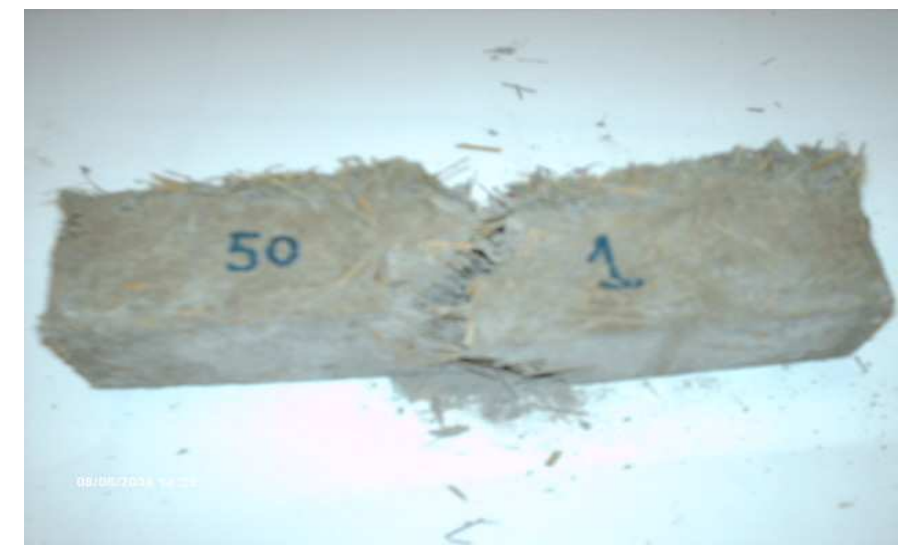

Figure 16 : Image de composite de diss non bovilli après essai de flexion. 


\section{Conclusion}

Les éprouvettes de fibres de diss bouilli ont pu résister à des contraintes de traction par essais de flexion assez considérables, comparées aux composites de fibres naturelles, sans qu'il ait apparition de fissures aux premières charges de rupture. En compression, les ruptures des éprouvettes de diss bouilli n'ont pas manifesté de rupture fragile, les éprouvettes sont restées presque intactes. Par contre les composites de fibres de diss naturels non traités n'ont manifesté aucune résistance, avec une rupture fragile, à cause de la présence des sucres évalués à plus $62 \%$ des composants hydrosolubles.

On peut retenir de ce travail les résultats suivants :

- Le traitement à l'eau bouillie a bien amélioré les caractéristiques mécaniques des composites alors que la structure de la fibre n'a pas évolué. Les fibres se placent horizontalement dans le composite, ce qui lui offre la capacité de travailler comme renforcement, sans pour autant augmenter les masses volumiques des composites soit 800 à $1200 \mathrm{~kg} / \mathrm{m} 3$.

- L'utilisation de ces composites comme remplissage dans les structures soumises aux efforts sismiques, qui sont réduits en raison de la légèreté du matériau, qui de plus présente un comportement ductile.

- Les améliorations effectivement apportées par la présence de fibres dans le composite sont dues essentiellement :

* au pouvoir adhérent des fibres à la pâte de ciment, à cause de leur structure épineuse,

* à la bonne résistance à la traction, due à la formation de " paquets ॥ par la pâte de ciment

* à leur structure fine leur permettant de se placer horizontalement, et ainsi de mieux participer à la reprise des efforts de traction.

\section{Références}

[1] - Jr. H. Savastano, P. G Warden, R. S. Coutts P, Brazilian waste fibres as reinforcement for cement-based composites, Cement Concrete Composites 22 (2000) 379-384.

[2] - A. Kriker, G. Debicki, A. Bali, M. M. Khenfer, M Chabannet, "Mechanical properties of date palm fibres and concrete reinforced with date palm fibres in hot-dry climate", Cement Concrete Composites 27(2005) 554-564. 
[3] - A. Ledhem, R. M. Dheilly, M. L. Benmalek, Quéneudec M, "Properties of Woodbased composites formulated with aggregate industry waste", Construction and Builiding Materials 14 (2000) 341-350

[4] - E. H. Aamr Daya, ॥Contribution à la valorisation de co-produits du lin, poussières obtenues par aspiration lors du teillage, dans une matrice cimentaire II, Thèse de doctorat de l'Université de Picardie Jules Verne., 2004.

[5] - P. Piementa, J. Chandellier, M. Rubaud, F. Dutruel, et H. Nicole, I Etude de la faisabilité des procédés à base de bétons de bois 11 , Cahier du CSTB 2703, Janvier-Février 1994.

[6] - Simatupang, Abbaureaktionen von Glucose, cellobiose und Holz unter dem Einfluss von Portlandzemzntmörtel, Holzfor schung, 40 pp 149-155, 1986.

[7] - M. C; Garci Juenger , H. M. Jennings, "New insights into the effects of sugar one the hydration and microstructure of cement pastes", Cement and Concrete Research 32 (2002) 393-399

[8] - K. Bilba, Arsene, M.A, Ouensanga, A, II Sugar cane bagasse fibre reinforced cement composites. Part I. Influence of the botanical components of bagasse on the setting of bagasse/cement composites", Cement Concrete Composites 25(2003) 91-96.

[9] - C. Asasutjarit, J. Hirunlabh, J. Khedari, S. Charoenvai, B. Zeghmati, Cheul Shin U, "Development of coconut coir-based lightweight cement board", Construction and Building Materials, 21 (2007) 277-288.

[10] - P; Costanlem, R; Jauberthie, F; Rendell, "Lightweight wood chipping concrete durability", Construction and Building Materials, 20(2006) 776-781.

[11] - V. K. Mathur, "Composite materials from local resources3, Construction and Building Materials, 20(2006) 470-477. 